\title{
Vertebral Artery Anomaly and Injury in Spinal Surgery
}

\author{
Robert Molinari $^{1} \quad$ Matthew Bessette ${ }^{1}$ Annie L. Raich ${ }^{2} \quad$ Joseph R. Dettori ${ }^{2}$ Christine Molinari ${ }^{1}$ \\ ${ }^{1}$ Department of Orthopaedic Surgery, University of Rochester Medical \\ Center, Rochester, New York, United States \\ 2 Spectrum Research, Inc., Tacoma, Washington, United States \\ Address for correspondence Robert Molinari, MD, Department of \\ Orthopaedic Surgery, University of Rochester Medical Center, 601 \\ Elmwood Avenue, Box 665, Rochester, NY 14642, United States \\ Evid Based Spine Care J 2014;5:16-27. \\ (e-mail: bobmol@aol.com; kan@rochester.rr.com).
}

\begin{abstract}
Study Design Systematic review.

Study Rationale The purpose of this review is to further define the published literature with respect to vertebral artery (VA) anomaly and injury in patients with degenerative cervical spinal conditions.

Objectives In adult patients with cervical spine or degenerative cervical spine disorders receiving cervical spine surgery, what is the incidence of VA injury, and among resulting VA injuries, which treatments result in a successful outcome and what percent are successfully repaired?

Materials and Methods A systematic review of pertinent articles published up to April 2013. Studies involving traumatic onset, fracture, infection, deformity or congenital abnormality, instability, inflammatory spinal diseases, or neoplasms were excluded. Two independent reviewers assessed the level of evidence quality using the Grades of Recommendation Assessment, Development and Evaluation criteria; disagreements

\section{Keywords}

- vertebral artery

- vertebral artery anatomy

- classification systems

- vertebral artery injury

- complication

- iatrogenic

- intraoperative complication

- cervical spine surgery were resolved by consensus.

Results From a total of 72 possible citations, the following met our inclusion criteria and formed the basis for this report. Incidence of VA injuries ranged from 0.20 to $1.96 \%$. None of the studies reported using preoperative imaging to identify anomalous or tortuous VA. Primary repair and ligation were the most effective in treating VA injuries. Conclusion The incidence of VA injuries in degenerative cervical spinal surgery might be as high as $1.96 \%$ and is likely underreported. Direct surgical repair is the most effective treatment option. The most important preventative technique for VA injuries is preoperative magnetic resonance imaging or computed tomography angiographic imaging to detect VA anomalies. The overall strength of evidence for the conclusions is low.
\end{abstract}

\section{Study Rationale and Context}

There is a paucity of published literature addressing the topic of vertebral artery (VA) anomaly and VA injury in degenerative cervical spine surgery. The incidence and risk factors for VA injury in degenerative spinal surgical

received

June 25, 2013

accepted after revision

November 15, 2013

procedures have not been adequately defined. The purpose of this study is to review the existing literature and to provide an accurate description of the published literature regarding variation of the normal VA anatomy and injury to the VA during degenerative cervical spinal surgery.

(c) 2014 Georg Thieme Verlag KG Stuttgart · New York
DOI http://dx.doi.org/ 10.1055/s-0034-1366980. ISSN 1663-7976. 


\section{Objectives}

Key Question (KQ) 1: In studies of adult patients with conditions warranting cervical spine surgery, what formal classification systems of VA anomalies based on imaging studies exist, and are these classification systems reliable?

In adult patients with cervical spine or degenerative cervical spine disorders receiving cervical spine surgery:

KQ 2: What is the incidence of VA injury?

KQ 3: Among the resulting VA injuries, which treatments result in a successful outcome and what percent are successfully repaired?

\section{Materials and Methods}

Study design: Systematic review.

Search: The databases included PubMed, Cochrane, and National Guideline Clearinghouse Databases; bibliographies of key articles.

Dates searched: The data were searched through April 19, 2013.

Inclusion criteria: Patients 18 years or older with cervical spine, degenerative cervical spine disorders, or other conditions warranting cervical spine surgery.

Exclusion criteria: Studies in patients younger than 18 years, those involving traumatic onset, cervical fracture, infection, deformity or congenital abnormality, instability, inflammatory spinal diseases, or neoplasms; case reports, comparative studies with fewer than 10 patients per treatment group; cadaveric studies; nonhuman in vivo, in vitro, and biomechanical studies.

Outcomes: Classification systems of VA anomalies; incidence of VA injuries; and successful outcome of VA injury treatment (no residual neurologic or vascular symptoms, including stroke, pain, neurologic deficit, or death).

Analysis: Descriptive statistics.

Overall strength of evidence: Risk of bias for individual studies was based on using criteria set by The Journal of Bone and Joint Surgery ${ }^{1}$ modified to delineate criteria associated with methodological quality and risk of bias based on recommendation from the Agency for Healthcare Research and Quality (AHRQ). ${ }^{2,3}$ The overall strength of evidence across studies was based on precepts outlined by the Grades of Recommendation Assessment, Development and Evaluation Working Group ${ }^{4}$ and recommendations made by the AHRQ. ${ }^{2,3}$

Details about methods can be found in online supplementary material.

\section{Results}

A total of 696 unique citations were retrieved for all three KQs. From a total of 72 citations evaluated for full-text review, 15 met the inclusion criteria for this report (-Fig. 1).

KQ 1: Formal Classification Systems of VA Anomalies We identified three case series that presented a classification system or a definition of VA anomalies. ${ }^{5-7}$
- Eskander et al $(2010)^{5}$ classified VA anomalies for C2 to C7 into three categories: intraforminal (midline migration of the VA, including tortuosity), extraforaminal (VA not contained within transverse foramen from C6 to C2), and arterial (VA that is fenestrated, hypoplastic, or absent). Eskander et al also suggested assessing VA abnormalities on magnetic resonance imaging (MRI) rather than plain radiograph and proposed a modification to Oga's classification for C1 to C7 VA tortuosity to account for arterial abnormalities (classified as normal or abnormal).

- Hong et al (2008) ${ }^{6}$ described anomalies of the V2 segment of the VA as: abnormal entrance (entrance into C4, C5, or C7 transverse foramen), V2 segment anomalies (bilateral or unilateral), and other variations of the VA (fenestrations or foraminal erosions).

- Oga et al (1996) ${ }^{7}$ classified the tortuosity of the VA from C1 to C7 into four categories based on the VA's relationship to three zones of the cervical spine. The categories are as follows: type 1 (straight and within zone I), type 2 (mildly tortuous and in zone I), type 3 (loop formation and most medial portion in zone II), and type 4 (loop formation and migration in zone III). Refer to - Table 1 for definitions of these zones.

None of the included studies conducted a formal analysis of the reliability of the proposed classification systems.

\section{KQ 2: Incidence of VA Injuries}

Incidence of VA injuries resulting from degenerative cervical spinal surgery ranged from 0 to $1.96 \%$ as reported in 12 studies. A total of $10 \mathrm{VA}$ injuries were reported ( - Tables 2 and 3).

- One RCT ( $\mathrm{CoE}$ II), ${ }^{8}$ three retrospective cohort studies ( $\mathrm{CoE}$ III), ${ }^{9-11}$ and eight case series (CoE IV) $)^{12-19}$ reported the incidence of VA injuries resulting from cervical spinal surgery. One of these 12 studies involved a posterior surgical procedure. ${ }^{19}$ Populations were predominantly male and middle aged. Sample sizes ranged from 20 to 1,976. Further details on the class of evidence rating for these studies can be found in the online supplementary material.

- Incidence was highest overall in corpectomy procedures in three studies $\left(1.96,{ }^{9} 1.41,{ }^{10}\right.$ and $0.20 \%{ }^{14}$ ) and uncoforaminotomy in one study $(1.11 \%){ }^{10}$

- The left VA was injured in the majority of cases $(86 \%, 6 / 7)$ in two studies reporting this level of detail. Burke et al $(2005)^{14}$ reported that five of the six VA injuries occurred in the left VA during corpectomy $(n=3)$ or anterior cervical discectomy and fusion $(n=2)$. Bilbao et al $(2010)^{13}$ reported that the one reported VA injury occurred in the left VA.

- The majority of VA injuries were detected intraoperatively $(80 \%, 8 / 10)^{9}, 10,13,14$ Only one VA injury was detected postoperatively $(10 \%, 1 / 10)$ in one study when the patient awoke with a lateral medullary infarct; subsequent cerebral angiography demonstrated a VA injury with posterior inferior cerebellar artery occlusion. ${ }^{14}$ Another study did 
2. Title/Abstract exclusion

Key question $1 \quad(n=147)$

Key question $2 \quad(n=228)$

Key question $3 \quad(n=358)$

\section{Total Citations}

Key question $1 \quad(n=161)$

Key question $2 \quad(n=274)$

Key question $3 \quad(n=358)$

\section{Excluded at full-text review \\ Key question $1 \quad(n=11)$ \\ Key question $2 \quad(n=34)$ \\ Key question 3 ( $n=9$ )}

Fig. 1 Flow chart showing results of literature search.

not report the diagnostic workup of the VA injury $(10 \%$, $1 / 10) .^{15}$

- None of the studies reported the phase of surgery resulting in the VA injury.

- None of the studies reported on the use of preoperative computed tomography (CT) or MRI to identify anomalous or tortuous VAs in their studies.

\section{KQ 3: Treatment of VA Injuries and Successful Outcomes}

No studies were identified that specifically addressed successful outcomes from the treatment for VA injuries. However, three studies identified from KQ 2 addressed the treatment of VA injuries. ${ }^{9,13,14}$ Overall, 63\% (5/8) of the VA injuries were successfully repaired (-Table 4).

- Primary repair and ligation were effective in treating VA injuries. $^{9,14}$

- Two successful primary repairs were reported by Burke et al (2005). ${ }^{14}$

- Lu et al $(2008)^{9}$ and Burke et al (2005) ${ }^{14}$ each reported one case of VA injury in which ligation repair was successful.

- Mixed results were reported using tamponade, resulting in only one successful repair in one study.

- One successful VA injury repair using tamponade was reported by Burke et al (2005). ${ }^{14}$
- Burke et al also reported one case of VA injury repaired by intraoperative tamponade. This patient suffered from hemodynamic instability from hypovolemia and subsequently died.

- A third VA injury was not detected until the patient suffered a postoperative lateral medullary infarct. The patient had received intraoperative tamponade for epidural oozing. Postoperative cerebellar angiography identified a VA injury with a posterior inferior cerebellar artery occlusion. This patient then received postoperative anticoagulation therapy.

- Embolization was attempted to repair one case of VA injury. ${ }^{13}$ The patient suffered multiple medullar, cerebellar, and supratentorial infarcts; at 18-month follow-up, the patient was ambulatory with aid.

- Anticoagulation therapy or no treatment: no studies reported these treatments.

\section{Clinical Guidelines}

None found.

\section{Evidence Summary}

The reported risk of VA in degenerative cervical spine surgery ranged from 0 to $2 \%$. The overall strength of evidence for determining risk is low; that is, we have low confidence that the evidence reflects the true risk. Regarding treatments for 
Table 1 Studies reporting classification systems of VA anomalies (KQ 1)

\begin{tabular}{|c|c|c|c|c|}
\hline $\begin{array}{l}\text { Author (y) } \\
\text { Study design }\end{array}$ & $\begin{array}{l}\text { Demographics } \\
\text { Diagnosis }\end{array}$ & $\begin{array}{l}\text { Classification system of } \\
\text { VA anomaly }\end{array}$ & $\begin{array}{l}\text { Method of assessing VA } \\
\text { anomaly }\end{array}$ & Notes \\
\hline $\begin{array}{l}\text { Eskander } \\
\text { et al }(2010) \\
\text { Case series }\end{array}$ & $\begin{array}{l}\text { - } N=250 \text { patients } \\
\text { - Age (mean): } 49 \text { y }(9-88) \\
\text { - Male: } 45 \% \\
\text { - Axial neck pain, } \\
\text { radiculopathy, } \\
\text { myelopathy }(n=N R)\end{array}$ & $\begin{array}{l}\text { VA anomalies for C2-C7: } \\
\text { - Intraforaminal } \\
\text { abnormalities: midline } \\
\text { migration with VA } \\
\text { located either medial to } \\
\text { or < } 1.5 \mathrm{~mm} \text { lateral to } \\
\text { the uncovertebral joint } \\
\text { - Extraforaminal } \\
\text { abnormalities: VA not } \\
\text { contained within } \\
\text { respective transverse } \\
\text { foramen from C6 to C2 } \\
\text { - Arterial abnormalities: } \\
\text { fenestrated, hypoplastic, } \\
\text { or absent }\end{array}$ & $\begin{array}{l}\text { - MRI from base of skull } \\
\text { thru T2, measurements } \\
\text { obtained from C2 to C7 } \\
\text { - IVAD } \\
\text { - MVAD } \\
\text { - UJVA } \\
\text { - VAD }\end{array}$ & $\begin{array}{l}\text { Author suggests modify- } \\
\text { ing Oga's classification for } \\
\text { VA tortuosity to account } \\
\text { for arterial abnormalities } \\
\text { and to assess the VA on } \\
\text { MRI, not radiograph. The } \\
\text { following is a proposed } \\
\text { addition to Oga's classifi- } \\
\text { cation: } \\
\text { - "A" for normal VA } \\
\text { ( } \leq 2 \mathrm{~mm} \text { size } \\
\text { differential) } \\
\text {-B" for abnormal VA } \\
\text { (hypoplastic/absent or } \\
\text { fenestrated arteries) }\end{array}$ \\
\hline $\begin{array}{l}\text { Hong et al (2008) } \\
\text { Case series }\end{array}$ & $\begin{array}{l}\text { - } N=350 \text { patients } \\
\text { (700 VA) } \\
\text { - Age (mean): } 54.8 \text { y } \\
\text { (16-89) } \\
\text { - Male: } 39 \% \\
\text { - Included: patients who } \\
\text { underwent CT } \\
\text { angiography for reasons } \\
\text { other than VA disease } \\
\text { - Excluded: bony abnor } \\
\text { malities (Klippel-Feil } \\
\text { syndrome and ankylosing } \\
\text { spondylitis) and VA } \\
\text { aplasia }\end{array}$ & $\begin{array}{l}\text { V2 segment of VA: } \\
\text { - Abnormal entrance: } \\
\text { entrance into C4, C5, or } \\
\text { C7 transverse foramen } \\
\text { - V2 segment anomalies: } \\
\text { bilateral and unilateral } \\
\text { - Variations of VA, including } \\
\text { fenestrations or foraminal } \\
\text { erosions }\end{array}$ & - CT angiography & $\begin{array}{l}\text { Author also includes } \\
\text { measurements between } \\
\text { the extraosseous portions } \\
\text { of the VA to surgical } \\
\text { landmarks }\end{array}$ \\
\hline $\begin{array}{l}\text { Oga et al (1996) } \\
\text { Case series }\end{array}$ & $\begin{array}{l}\text { - } N=23 \text { patients } \\
\text { - Cervical myelopathy from } \\
\text { cervical spondylosis or } \\
\text { cervical disc herniation } \\
(n=23)\end{array}$ & $\begin{array}{l}\text { VA tortuosity for C1-C7: } \\
\text { - Type 1: VA straight and } \\
\text { in zone I } \\
\text { - Type 2: mildly tortuous } \\
\text { and in zone I } \\
\text { - Type 3: loop formation } \\
\text { and most medial portion } \\
\text { in zone II } \\
\text { - Type 4: loop formation } \\
\text { and migration in zone III }\end{array}$ & $\begin{array}{l}\text { - Radiograph, MRI of } \\
\text { C1-C7, vertebral } \\
\text { arteriography, or MRI } \\
\text { angiography, CT scan }\end{array}$ & \\
\hline
\end{tabular}

Abbreviations: $C T$, computed tomography; KQ, key question; IVAD, intervertebral artery distance; MRI, magnetic resonance imaging; MVAD, midline VA distance; UJVA, uncovertebral joint VA distance; VA, vertebral artery; VAD, VA diameter.

${ }^{a} \mathrm{~V} 2$ segment defined as being between C5 and C2 transverse process (Hong et al, 2008); zones from the anteroposterior view for VA tortuosity defined as: zone I (outside of the lateral end of Luschka joint), zone II (between the lateral and medial end of Luschka joint), and zone III (medial of Luschka joint) (Oga et al, 1996).

VA injuries, three studies reported 5/8 (63\%) successful repairs. The overall strength of evidence for this estimate is low (-Table 5).

\section{Illustrative Case}

A 69-year-old man presented with a 3-month history of progressive cervical myelopathy (-Fig. 2). The patient's preoperative MRI and CT studies demonstrated an abnormal posterior course of the right VA (-Figs. 3 and 4 ). Injury of this anomalous VA segment occurred during posterior surgical exposure. Intraoperative direct repair of the injury was performed by the on-call vascular surgeon using 6.0 Prolene sutures (Ethicon, Somerville, NJ, USA) (-Fig. 5). The patient had an uneventful postoperative course without clinical evidence of VA injury. He demonstrated excellent recovery from his clinical myelopathy at 6 months postoperative.

\section{Discussion}

- This systematic review is limited by the following:

- The majority of studies were CoE III or IV.

- None of the three classification systems of VA anomalies found in the literature was validated.

- Some of the studies reporting on VA injuries had a relatively small sample size ( $<100$ patients). Given the rarity of VA injuries, larger study populations are needed to identify this surgical complication.

- This review highlights several important issues with respect to VA injury in degenerative spinal surgery. Most important is that the incidence of this potentially devastating complication may be as high as $1.96 \%$ of all cases performed, which should serve to raise the level of awareness among spinal surgeons. 
20 Vertebral Artery Anomaly and Injury in Spinal Surgery Molinari et al.

Table 2 Studies reporting frequencies for and outcomes of VA injuries resulting from cervical spine surgery (KQs 2 and 3)

\begin{tabular}{|c|c|c|c|c|c|}
\hline $\begin{array}{l}\text { Author (y) } \\
\text { Study design } \\
\text { CoE }\end{array}$ & $\begin{array}{l}\text { Demographics } \\
\text { Diagnosis }\end{array}$ & Type of surgery & $\begin{array}{l}\text { Incidence of VA } \\
\text { injuries }^{\mathrm{b}} \\
\text { VA affected }^{-}\end{array}$ & $\begin{array}{l}\text { Diagnosis, treat- } \\
\text { ment, and outcome } \\
\text { from VA injury }\end{array}$ & $\begin{array}{l}\text { Potential risk } \\
\text { factors for VA injury }\end{array}$ \\
\hline \multicolumn{6}{|l|}{ Anterior procedures } \\
\hline $\begin{array}{l}\text { Bilbao et al (2010) } \\
\text { Case series } \\
\text { CoE: IV } \\
\text { (KQs } 2 \text { and } 3 \text { ) }\end{array}$ & $\begin{array}{l}\text { - } N=71 \\
\text { - Age (mean): NR (> } \\
50 \text { y: } n=48 \\
<50 \text { y: } n=23) \\
\text { - Male: } 63 \% \\
\text { - Spondylotic spinal } \\
\text { cord compression } \\
\text { with tetraparesis } \\
(n=29), \text { pain } \\
(n=21), \text { motor } \\
\text { paresis }(n=12), \\
\text { and paresthesia } \\
(n=9)\end{array}$ & $\begin{array}{l}\text { Corpectomy with ti- } \\
\text { tanium mesh or } \\
\text { telescopic cage and } \\
\text { autograft: } \\
\text { - } 1 \text { level }(n=46), 2 \\
\text { levels }(n=25) \\
\text { - right-side ap- } \\
\quad \text { proach }(n=71)\end{array}$ & $\begin{array}{l}\text { - } 1.4 \%(1 / 71) \\
\text { - VA affected: left } \\
(n=1)\end{array}$ & $\begin{array}{l}\text { Patient 1: } \\
\text { - Intraoperative di- } \\
\text { agnosis (details } \\
\text { NR). Treatment: } \\
\text { embolization } \\
\text { above and below } \\
\text { damaged site. } \\
\text { Outcome: multiple } \\
\text { medullar, cerebel- } \\
\text { lar, and supraten- } \\
\text { torial infarcts; at } \\
18 \text { mo patient is } \\
\text { ambulatory with } \\
\text { aid and has normal } \\
\text { deglutition }\end{array}$ & \\
\hline $\begin{array}{l}\text { Lu et al (2008) } \\
\text { Retrospective cohort } \\
\text { CoE: III } \\
\text { (KQs } 2 \text { and 3) }\end{array}$ & $\begin{array}{l}\text { - } N=51 \\
\text { - Age (mean): } 61.0 \text { y } \\
\text { ( } 37-86) \\
\text { - Male: } 75 \% \\
\text { - Multilevel degen } \\
\text { erative cervical } \\
\text { myelopathy with } \\
\text { upper extremity } \\
\text { paresis, walking } \\
\text { and balance } \\
\text { problems, pro } \\
\text { gressive } \\
\text { myelopathy, } \\
\text { persistent } \\
\text { radicular pain } \\
\text { Aged group: } n=20, \\
\text { mean age } 75 \text { y (70- } \\
\text { 86) } \\
\text { Control group: } n=31 \text {, } \\
\text { mean age } 52 \text { y (37- } \\
68)\end{array}$ & $\begin{array}{l}\text { Corpectomy with ti- } \\
\text { tanium mesh and } \\
\text { autograft }\end{array}$ & $\begin{array}{l}\text { - } 1.96 \%(1 / 51) \\
\text { - VA affected: NR }\end{array}$ & $\begin{array}{l}\text { Patient 1: } \\
\text { - Intraoperative di- } \\
\text { agnosis (details } \\
\text { NR). Treatment: li- } \\
\text { gation. Outcome: } \\
\text { patient recovered } \\
\text { after blood } \\
\text { transfusion }\end{array}$ & $\begin{array}{l}\text { VA injury occurred in } \\
\text { one patient in the } \\
\text { aged group }\end{array}$ \\
\hline $\begin{array}{l}\text { Pechlivanis et al } \\
\text { (2008) } \\
\text { Retrospective cohort } \\
\text { CoE: III } \\
\text { (KQ } 2 \text { only) }\end{array}$ & $\begin{array}{l}\text { - } N=90 \\
\text { - Age (mean): } \\
47.9 \pm 8.7 \mathrm{y} \\
(29-69) \\
\text { - Male: } 50 \% \\
\text { - Cervical degenera- } \\
\text { tive disc disease } \\
\text { with soft-, hard-, or } \\
\text { soft/hard-disc pa- } \\
\text { thology } \\
\text { Soft-disc group: } \\
n=49, \text { mean age } \\
47.9 \text { y (29-69) } \\
\text { Hard-disc group: } \\
n=24, \text { mean age } \\
47.8 \text { y (36-65) } \\
\text { Soft/hard-disc group: } \\
n=17, \text { mean age } \\
46.7 \text { y (41-65) }\end{array}$ & $\begin{array}{l}\text { Single-level uncofor- } \\
\text { aminotomy using Jho } \\
\text { technique with Sar- } \\
\text { inger modification }\end{array}$ & $\begin{array}{l}\text { - } 1.11 \%(1 / 90) \\
\text { - VA affected: NR }\end{array}$ & $\begin{array}{l}\text { Patient 1: } \\
\text { - Intraoperative di- } \\
\text { agnosis (details } \\
\text { NR, surgery dis- } \\
\text { continued). } \\
\text { Treatment: NR. } \\
\text { Outcome: no oc- } \\
\text { clusion docu- } \\
\text { mented on } \\
\text { postoperative an- } \\
\text { giography, no } \\
\text { neurological defi- } \\
\text { cits experienced, } \\
\text { successful reoper- } \\
\text { ation at } 3 \text { mo }\end{array}$ & $\begin{array}{l}\text { The following tech- } \\
\text { niques were used to } \\
\text { protect the VA: the } \\
\text { Saringer surgical } \\
\text { modification was } \\
\text { used, drilling was } \\
\text { conducted using a } \\
\text { diamond drill, and a } \\
\text { thin layer of the cor- } \\
\text { tical bone of the lat- } \\
\text { eral wall of the } \\
\text { uncinate process was } \\
\text { preserved. } \\
\text { VA injury occurred in } \\
\text { one patient in the } \\
\text { hard-disc pathology } \\
\text { group }\end{array}$ \\
\hline $\begin{array}{l}\text { Choi et al (2007) } \\
\text { Case series } \\
\text { CoE: IV } \\
\text { (KQ } 2 \text { only) }\end{array}$ & $\begin{array}{l}\text { - } N=20 \\
\text { - Age (mean): } 48.7 \text { y } \\
\text { ( } 37-74) \\
\text { - Male: } 75 \% \\
\text { - Cervical radiculop- } \\
\text { athy from soft disc } \\
\text { herniation }(n=7), \\
\text { spondylotic stenosis } \\
\text { with osteophytes } \\
\text { ( } n=3), \text { soft disc } \\
\text { with osteophytes } \\
(n=10)\end{array}$ & $\begin{array}{l}\text { Modified transcor- } \\
\text { poreal anterior cervi- } \\
\text { cal microforaminot- } \\
\text { omy (transverse skin } \\
\text { incision made at } 1 \\
\text { level higher than af- } \\
\text { fected disc level) }\end{array}$ & $0 \%(0 / 20)$ & $\mathrm{n} / \mathrm{a}$ & $\begin{array}{l}\text { Author claims that } \\
\text { with this technique } \\
\text { VA artery is not } \\
\text { exposed or } \\
\text { endangered }\end{array}$ \\
\hline
\end{tabular}


Table 2 (Continued)

\begin{tabular}{|c|c|c|c|c|c|}
\hline $\begin{array}{l}\text { Author (y) } \\
\text { Study design } \\
\text { CoE }\end{array}$ & $\begin{array}{l}\text { Demographics } \\
\text { Diagnosis }\end{array}$ & Type of surgery & $\begin{array}{l}\text { Incidence of VA } \\
\text { injuries }^{\mathrm{b}} \\
\text { VA affected }\end{array}$ & $\begin{array}{l}\text { Diagnosis, treat- } \\
\text { ment, and outcome } \\
\text { from VA injury }\end{array}$ & $\begin{array}{l}\text { Potential risk } \\
\text { factors for VA injury }\end{array}$ \\
\hline $\begin{array}{l}\text { Fountas et al (2007) } \\
\text { Case series } \\
\text { CoE: IV } \\
\text { (KQ } 2 \text { only) }\end{array}$ & $\begin{array}{l}\text { - } N=1,015 \\
\text { - Age (mean): } 56.3 \text { y } \\
\text { (28-75) } \\
\text { - Male: } 54 \% \\
\text { - Cervical radiculop- } \\
\text { athy and/or mye- } \\
\text { lopathy secondary } \\
\text { to degenerative disc } \\
\text { disease and/or cer- } \\
\text { vical spondylosis }\end{array}$ & $\begin{array}{l}\text { ACDF: } \\
\text { - Right-side ap- } \\
\text { proach } 93.8 \% \text {, } \\
\text { left-side approach } \\
6.2 \%\end{array}$ & $0 \%(0 / 1015)$ & $\mathrm{n} / \mathrm{a}$ & \\
\hline $\begin{array}{l}\text { Sasso et al (2007) } \\
\text { RCT } \\
\text { CoE: II } \\
\text { (KQ } 2 \text { only) }\end{array}$ & $\begin{array}{l}\text { - } N=115 \\
\text { - Age (mean): } 44.0 \text { y } \\
\text { ( } 25.1-66.9) \\
\text { - Male: } 54 \% \\
\text { - Single-level cervical } \\
\text { degenerative dis- } \\
\text { ease causing radi- } \\
\text { culopathy or } \\
\text { myelopathy } \\
\text { Bryan disc group: } \\
n=56, \text { mean age } \\
42.5 \pm 7.8 \text { y ( } 25.1- \\
64) \\
\text { ACDF group: } n=59 \text {, } \\
\text { mean age } \\
46.1 \pm 7.8 \text { y (29.4- } \\
66.9)\end{array}$ & $\begin{array}{l}\text { - Single-level } \\
\text { cervical } \\
\text { arthroplasty with } \\
\text { Bryan cervical } \\
\text { disc prosthesis } \\
(n=56) \\
\text { - Single-level ACDF } \\
\text { with allograft and } \\
\text { plate }(n=59)\end{array}$ & $0 \%(0 / 115)$ & $\mathrm{n} / \mathrm{a}$ & \\
\hline $\begin{array}{l}\text { Burke et al (2005) } \\
\text { Case series } \\
\text { CoE: IV } \\
\text { (KQs } 2 \text { and } 3 \text { ) }\end{array}$ & $\begin{array}{l}\text { - } N=1,976 \text { patients } \\
\text { - Age (mean): NR } \\
\text { - Male: \% NR } \\
\text { - Herniated or degen- } \\
\text { erative disc disease } \\
\text { or spondylosis }\end{array}$ & $\begin{array}{l}\text { Anterior cervical } \\
\text { spine procedures } \\
\text { (right-side approach) } \\
\text { with VA injuries oc- } \\
\text { curring during: } \\
\text { - ACDF, } 1 \text { level } \\
(n=1) \\
\text { - ACDF, } 3 \text { levels } \\
(n=1) \\
\text { - Anterior cervical } \\
\text { corpectomy + } \\
\text { fusion } \\
\text { reexploration } \\
(n=1) \\
\text { - Anterior cervical } \\
\text { corpectomy + } \\
\text { fusion }(n=3)\end{array}$ & $\begin{array}{l}-0.3 \%(6 / 1,976) \\
- \text { left VA }(n=5) \\
\text { right VA }(n=1)\end{array}$ & 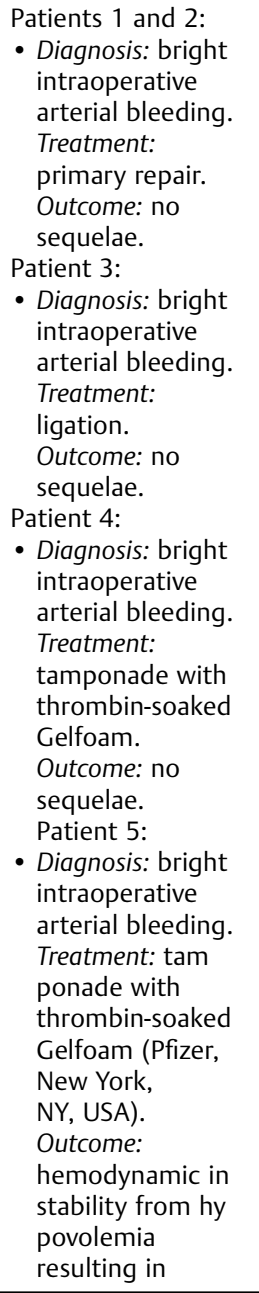 & $\begin{array}{l}\text { Operating micro- } \\
\text { scope used in } 83 \% \text { ( } 5 / \\
6 \text { ) of surgeries re- } \\
\text { sulting in VA injury. } \\
\text { Intraoperative CT } \\
\text { scan was not per- } \\
\text { formed in any of the } \\
\text { surgeries that in- } \\
\text { curred a VA injury }\end{array}$ \\
\hline
\end{tabular}


22 Vertebral Artery Anomaly and Injury in Spinal Surgery Molinari et al.

Table 2 (Continued)

\begin{tabular}{|c|c|c|c|c|c|}
\hline $\begin{array}{l}\text { Author }(y) \\
\text { Study design } \\
\text { CoE }\end{array}$ & $\begin{array}{l}\text { Demographics } \\
\text { Diagnosis }\end{array}$ & Type of surgery & $\begin{array}{l}\text { Incidence of VA } \\
\text { injuries }^{\mathrm{b}} \\
\text { VA affected }\end{array}$ & $\begin{array}{l}\text { Diagnosis, treat- } \\
\text { ment, and outcome } \\
\text { from VA injury }\end{array}$ & $\begin{array}{l}\text { Potential risk } \\
\text { factors for VA injury }\end{array}$ \\
\hline & & & & $\begin{array}{l}\text { intraoperative } \\
\text { death. } \\
\text { Patient 6: } \\
\text { - Diagnosis: intra- } \\
\text { operative epidural } \\
\text { oozing. Treatment: } \\
\text { tamponade with } \\
\text { thrombin-soaked } \\
\text { Gelfoam and post- } \\
\text { operative anticoa- } \\
\text { gulation with } \\
\text { heparin. Outcome: } \\
\text { posterior inferior } \\
\text { cerebellar artery } \\
\text { occlusion, lateral } \\
\text { medullary infarct, } \\
\text { and syndrome. }\end{array}$ & \\
\hline $\begin{array}{l}\text { Shen et al (2004) } \\
\text { Retrospective cohort } \\
\text { CoE: III } \\
\text { (KQ } 2 \text { only) }\end{array}$ & $\begin{array}{l}\text { - } N=109 \text { patients } \\
\text { - Age (mean): } 46.3 \text { y } \\
\text { (27-83) } \\
\text { - Male: } 60 \% \\
\text { - Radiculopathy sec- } \\
\text { ondary to herniated } \\
\text { disc and/or } \\
\text { spondylosis }\end{array}$ & $\begin{array}{l}\text { ACDF (Smith-Robin- } \\
\text { son left-side ap- } \\
\text { proach): } \\
\text { - Uncovertebral joint } \\
\text { decompression } \\
(n=71) \\
\text { - Indirect decom- } \\
\text { pression by means } \\
\text { of disc space dis- } \\
\text { traction }(n=38)\end{array}$ & $0 \%(0 / 109)$ & $\mathrm{n} / \mathrm{a}$ & \\
\hline $\begin{array}{l}\text { Graham et al (1996) } \\
\text { Case series } \\
\text { CoE: IV } \\
\text { (KQ } 2 \text { only) }\end{array}$ & $\begin{array}{l}\text { - } N=21 \text { patients } \\
\text { - Age (mean): } \\
52.4 \pm 15.2 \text { y }(28- \\
81) \\
\text { - Male: } 81 \% \\
\text { - Degenerative spon- } \\
\text { dylosis }(n=17), \\
\text { congenital stenosis } \\
(n=2), \text { trauma } \\
(n=2), \text { OPLL } \\
(n=1)\end{array}$ & $\begin{array}{l}\text { Cervical arthrodesis } \\
\text { and stabilization with } \\
\text { lateral mass plate } \\
\text { (anterior approach } \\
n=20 \text {, posterior } \\
\text { approach } n=1 \text { ) }\end{array}$ & $0 \%(0 / 21)$ & $\mathrm{n} / \mathrm{a}$ & $\begin{array}{l}17 \%(5 / 29) \text { screws } \\
\text { placed in central axial } \\
\text { zone were malposi- } \\
\text { tioned, placing the } \\
\text { VA at risk }\end{array}$ \\
\hline $\begin{array}{l}\text { Bertalanffy and Eg- } \\
\text { gert (1989) } \\
\text { Case series } \\
\text { CoE: IV } \\
\text { (KQ } 2 \text { only) }\end{array}$ & $\begin{array}{l}\text { - } N=450 \\
\text { - Age (mean): } 50 \text { y } \\
\text { ( } 25-78) \\
\text { - Male: } 72 \% \\
\text { - Cervical degenera- } \\
\text { tive disc disease } \\
\text { with radiculopathy } \\
\text { (61\%), pure myelop- } \\
\text { athy (16\%), or com- } \\
\text { bined myeloradicul- } \\
\text { opathy (23\%) }\end{array}$ & $\begin{array}{l}\text { Anterior cervical dis- } \\
\text { cectomy without } \\
\text { fusion }\end{array}$ & $0 \%(0 / 450)$ & $\mathrm{n} / \mathrm{a}$ & \\
\hline $\begin{array}{l}\text { Busch (1978) } \\
\text { Case series } \\
\text { CoE: IV } \\
\text { (KQ } 2 \text { only) }\end{array}$ & $\begin{array}{l}\text { - } N=138 \\
\text { - Age (mean): } 50.5 \text { y } \\
\text { ( } 27-74) \\
\text { - Male: } 61 \% \\
\text { - Cervical spondylosis } \\
\text { from: soft prolapse } \\
(n=49) \text {, segmental } \\
\text { deficits }(n=54), \\
\text { compression of spi- } \\
\text { nal cord }(n=29), \\
\text { amyotrophic lateral } \\
\text { sclerosis }(n=3), \\
\text { spinal muscular at- } \\
\text { rophy ( } n=1), \text { spas- } \\
\text { tic spinal paralysis } \\
\text { ( } n=1), \text { plexus car- } \\
\text { cinoma polyneurop- } \\
\text { athy }(n=1)\end{array}$ & $\begin{array}{l}\text { ACDF with ICBG or } \\
\text { Kiel bone graft }\end{array}$ & $\begin{array}{l}\text { - } 0.7 \% \text { ( } 1 / 138) \\
\text { - VA affected: NR }\end{array}$ & $\begin{array}{l}\text { Patient 1: } \\
\text { - Diagnosis: NR. } \\
\text { Treatment: NR. } \\
\text { Outcome: death }\end{array}$ & $\begin{array}{l}\text { Author reports that } \\
\text { VA injury occurred in } \\
\text { an atypical VA, but } \\
\text { no other details were } \\
\text { given }\end{array}$ \\
\hline
\end{tabular}


Table 2 (Continued)

\begin{tabular}{|c|c|c|c|c|c|}
\hline $\begin{array}{l}\text { Author (y) } \\
\text { Study design } \\
\text { CoE }\end{array}$ & $\begin{array}{l}\text { Demographics }{ }^{a} \\
\text { Diagnosis }\end{array}$ & Type of surgery & $\begin{array}{l}\text { Incidence of VA } \\
\text { injuries }^{\mathbf{b}} \\
\text { VA affected }\end{array}$ & $\begin{array}{l}\text { Diagnosis, treat- } \\
\text { ment, and outcome } \\
\text { from VA injury }\end{array}$ & $\begin{array}{l}\text { Potential risk } \\
\text { factors for VA injury }\end{array}$ \\
\hline \multicolumn{6}{|l|}{ Posterior procedures } \\
\hline $\begin{array}{l}\text { Katonis et al (2011) } \\
\text { Case series } \\
\text { CoE: IV } \\
\text { (KQ } 2 \text { only) }\end{array}$ & $\begin{array}{l}\text { - } N=225 \\
\text { - Age (mean): } 68 \text { y } \\
(45-84) \\
\text { - Male: } 53 \% \\
\text { - Cervical spondylosis } \\
\text { with myelopathy } \\
(n=225)\end{array}$ & $\begin{array}{l}\text { Posterior cervical fix- } \\
\text { ation using screw- } \\
\text { plate and polyaxial } \\
\text { screw-rod implant } \\
\text { system }\end{array}$ & $0 \%(0 / 225)$ & $\mathrm{n} / \mathrm{a}$ & $\begin{array}{l}\text { Suboptimal screw } \\
\text { placement present in } \\
0.6 \%(11 / 1,662 \\
\text { screws), but did not } \\
\text { result in VA injury }\end{array}$ \\
\hline
\end{tabular}

Abbreviations: ACDF, anterior cervical discectomy and fusion; CT, computed tomographic scan; ICBG, iliac crest bone graft; KQ, key question; n/a, not applicable; NR, not reported; OPLL, ossification of the posterior longitudinal ligament; RCT, randomized controlled trial; VA, vertebral artery. ${ }^{a}$ Demographics for patients with 24-month follow-up (Pechlivanis et al, 2008) or minimum 12-month follow-up (Choi et al, 2007).

${ }^{b}$ Author reports $0.76 \%$ incidence of VA injuries, but it appears that intraoperative findings are presented for 90 patients with 24 -month follow-up (Pechlivanis et al, 2008).

Table 3 Studies reporting frequencies for VA injuries resulting from cervical spine surgery

\begin{tabular}{|c|c|}
\hline Surgical procedure & VA injury, \% (n/N) \\
\hline \multicolumn{2}{|l|}{ Anterior procedures } \\
\hline \multicolumn{2}{|l|}{ ACDF } \\
\hline Fountas et al (2007) & $0 \%(0 / 1015)$ \\
\hline Burke et al $(2005)^{a}$ & $0.10 \%(2 / 1,976)$ \\
\hline Shen et al (2004) & $0 \%(0 / 109)$ \\
\hline Busch (1978) & $0.72 \%(1 / 138)$ \\
\hline \multicolumn{2}{|l|}{ ACDF or cervical arthroplasty } \\
\hline Sasso et al (2007) & $0 \%(0 / 115)$ \\
\hline \multicolumn{2}{|c|}{ Cervical arthrodesis/stabilization with lateral mass plate } \\
\hline Graham et al (1996) & $0 \%(0 / 21)$ \\
\hline \multicolumn{2}{|l|}{ Cervical discectomy without fusion } \\
\hline Bertalanffy and Eggert (1989) & $0 \%(0 / 450)$ \\
\hline \multicolumn{2}{|l|}{ Corpectomy } \\
\hline Bilbao et al (2010) & $1.41 \%(1 / 71)$ \\
\hline Lu et al (2008) & $1.96 \%(1 / 51)$ \\
\hline Burke et al (2005), primary or re-explorationa & $0.20 \%(4 / 1,976)$ \\
\hline \multicolumn{2}{|l|}{ Modified transcorporeal microforaminotomy } \\
\hline Choi et al (2007) & $0 \%(0 / 20)$ \\
\hline \multicolumn{2}{|l|}{ Uncoforaminotomy } \\
\hline Pechlivanis et al (2008) & $1.11 \%(1 / 90)$ \\
\hline \multicolumn{2}{|l|}{ Posterior procedures } \\
\hline \multicolumn{2}{|c|}{ Cervical fixation using screw-plate/polyaxial screw-rod implant } \\
\hline Katonis et al (2011) & $0 \%(0 / 225)$ \\
\hline
\end{tabular}

Abbreviations: ACDF, anterior cervical discectomy and fusion; VA, vertebral artery.

${ }^{a}$ Author does not report the types of specific anterior cervical spine procedures included in the study other than those in which a VA injury occurred (Burke et al, 2005). 
24 Vertebral Artery Anomaly and Injury in Spinal Surgery Molinari et al.

Table 4 Treatments and success rates for VA injuries resulting from cervical spine surgery

\begin{tabular}{|l|l|l|}
\hline Treatment for VA injury & $\begin{array}{l}\text { No. of patients } \\
\text { receiving treatment }\end{array}$ & $\begin{array}{l}\text { \% patients with } \\
\text { successful outcome }\end{array}$ \\
\hline Primary repair (Burke et al, 2005) & 2 & $100 \%$ \\
\hline Ligation (Lu et al, 2008; Burke et al, 2005) & 2 & $100 \%$ \\
\hline Tamponade (Burke et al, 2005) & 2 & $50 \%$ \\
\hline Tamponade with postoperative anticoagulation (Burke et al, 2005) & 1 & $0 \%$ \\
\hline Embolization (Bilbao et al, 2010) & 1 & $0 \%$ \\
\hline Anticoagulation only & 0 & n/a \\
\hline No treatment & 0 & n/a \\
\hline
\end{tabular}

Abbreviations: $\mathrm{n} / \mathrm{a}$, not applicable; VA, vertebral artery.

${ }^{\mathrm{a}}$ Two studies reported the outcome from treatment of VA injury (successful outcome in one patient (Pechlivanis et al, 2008), death in one patient (Busch, 1978), but did not report the treatment administered.

bSuccessful outcome defined as: no residual neurologic or vascular symptoms, including stroke, pain, neurologic deficit, or death.

\section{Table 5 Evidence summary}

Baseline quality: High = majority of articles level I/II; low = majority of articles level III/IV Upgrade: Large magnitude of effect (1 or 2 classes); dose response gradient (1 class) Downgrade: Inconsistency of results (1 or 2 classes); indirectness of evidence (1 or 2 classes); imprecision of effect estimates ( 1 or 2 classes)

\begin{tabular}{|l|l} 
Strength of evidence & Conclusions/comments \\
\hline
\end{tabular}

KQ 1: In studies of adult patients with conditions warranting cervical spine surgery, what formal classification systems of VA anomalies based on imaging studies exist and are these classification systems reliable?

\begin{tabular}{|l|l|l|}
\hline $\begin{array}{l}\text { Reliability of } \\
\text { classification } \\
\text { systems }\end{array}$ & Not applicable & $\begin{array}{l}\text { None of the included studies conducted } \\
\text { a formal analysis of the reliability } \\
\text { of the proposed classification systems. }\end{array}$ \\
\hline
\end{tabular}

KQ 2: In adult patients receiving cervical spinal surgery, what is the incidence of VA injuries?

\begin{tabular}{|l|l|l|l|}
\hline $\begin{array}{l}\text { Incidence of VA } \\
\text { injuries }\end{array}$ & Low & $\begin{array}{l}\text { One RCT, three retrospective cohort studies, and } \\
\text { eight case series reported on VA injuries during } \\
\text { cervical spine surgery. Seven studies reported no } \\
\text { VA injuries. Five studies reported rates of } 0.10 \text { to } \\
1.96 \% \text {, depending on the type of anterior cervical } \\
\text { spine procedure. No VA injuries were reported in } \\
\text { the one study comprising posterior spine procedures. }\end{array}$ \\
\hline
\end{tabular}

KQ 3: Among VA injuries resulting from cervical spinal surgery in adult patients, which treatments result in successful outcome and what percent are successfully repaired?

\begin{tabular}{|l|l|l|l|}
$\begin{array}{l}\text { Treatments } \\
\text { resulting } \\
\text { in successful } \\
\text { outcome }\end{array}$ & $\mid \begin{array}{l}\text { Low } \\
\text { reported on VA injury treatments and treatment } \\
\text { outcome. Primary repair and ligation were effective in } \\
\text { treating VA injuries with patients experiencing no } \\
\text { residual neurologic or vascular symptoms. Mixed } \\
\text { results were seen with tamponade, with only one of } \\
\text { three cases resulting in successful outcome. } \\
\text { Embolization was attempted in one case and did not } \\
\text { result in a successful outcome. No studies reported } \\
\text { on anticoagulation therapy only or no treatment. }\end{array}$ \\
\hline
\end{tabular}

Abbreviations: CoE, class of evidence; KQ, key question; VA, vertebral artery.

Notes: All AHRQ "required" and "additional" domains are assessed. Only those that influence the baseline grade are listed in the table.

Baseline strength: Risk of bias (including control of confounding) is accounted for in the individual article evaluations. High = majority of articles level $\mathrm{I} / \mathrm{II}$; low = majority of articles level III/IV.

Downgrade: Inconsistency ${ }^{\mathbf{b}}$ of results (1 or 2); indirectness of evidence (1 or 2); imprecision of effect estimates (1 or 2); subgroup analyses not stated a priori and no test for interaction (2).

Upgrade: Large magnitude of effect (1 or 2); dose response gradient (1).

${ }^{a}$ Required domains: risk of bias, consistency, directness, precision. Plausible confounding that would decrease observed effect is accounted for in our baseline risk of bias assessment through individual article evaluation. Additional domains: dose-response, strength of association, publication bias.

bSingle study = "consistency unknown." 


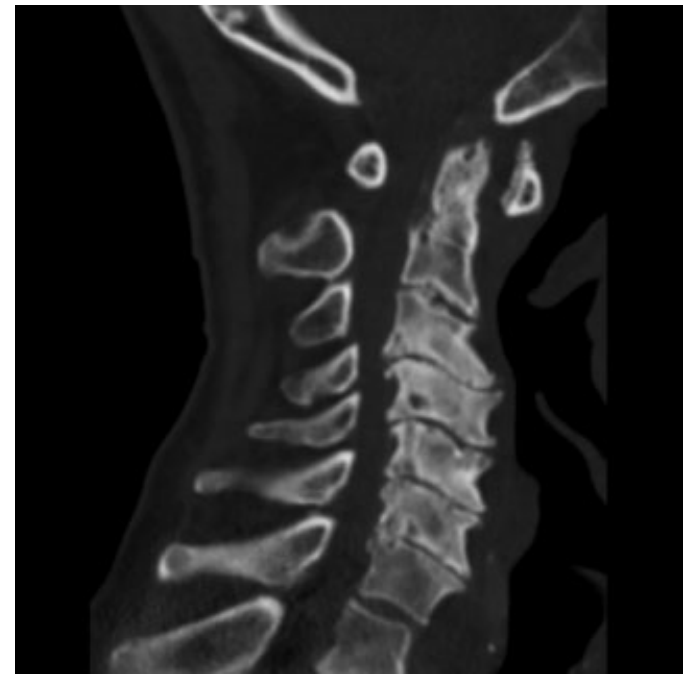

Fig. 2 Preoperative sagittal CT image of a 69-year-old man with multilevel cervical spinal stenosis, C1-2 instability, and progressive myelopathy. $\mathrm{CT}$, computed tomography.

- It is our opinion that the VA classification system described by Eskander et al (2010) is the most comprehensive and useful to surgeons for the purpose of preoperative planning. When applied to a preoperative MRI study, this classification system enables surgeons to detect and grade VA anomalies in an organized manner. Grading VA anatomy with respect to intraforaminal midline migration, extraforaminal abnormal entrance to the cervical spine, and arterial side dominance is clearly the most important preventative technique described to date and should become an integral portion of degenerative cervical spinal surgeons' preoperative routine.

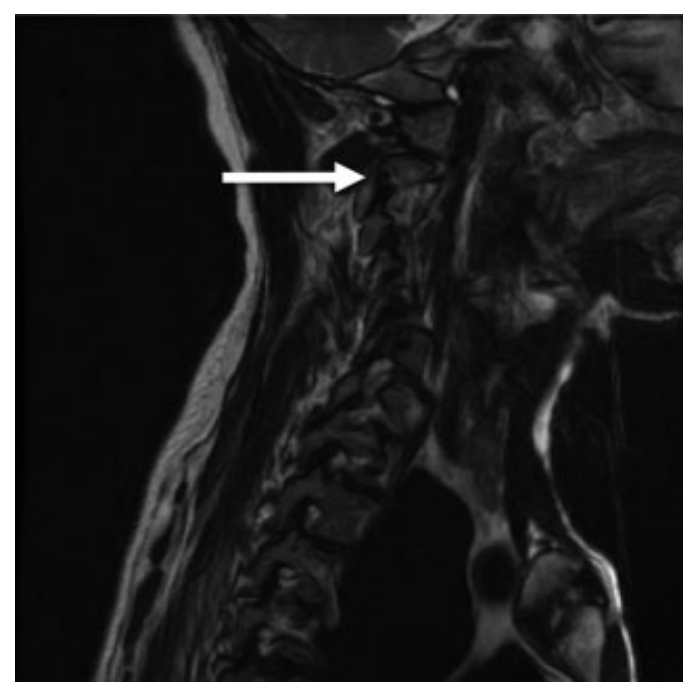

Fig. 3 Sagittal T2 MRI image showing abnormal posterior course of the right V2 VA segment. MRI, magnetic resonance imaging; VA, vertebral artery.

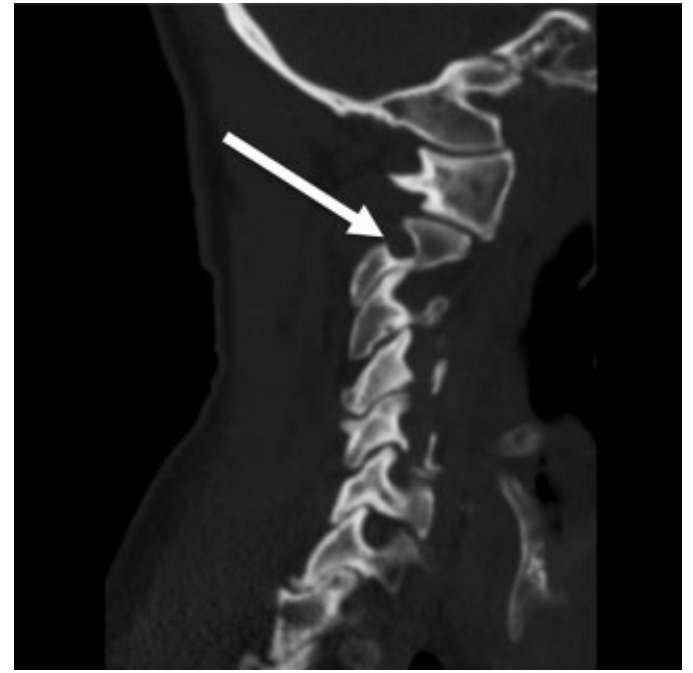

Fig. 4 Sagittal CT image showing abnormal posterior course of the right V2 VA segment. CT, computed tomography; VA, vertebral artery.

- While relatively few degenerative cervical surgery VA injuries have been reported, it is likely that the true incidence might be higher that what is currently depicted in the literature.

- It is clearly apparent from our review that when the intraoperative injury to the VA is encountered, direct surgical repair is the treatment option that results in the best clinical outcomes.

- There remains a paucity of existing literature on this topic and much more research is needed.

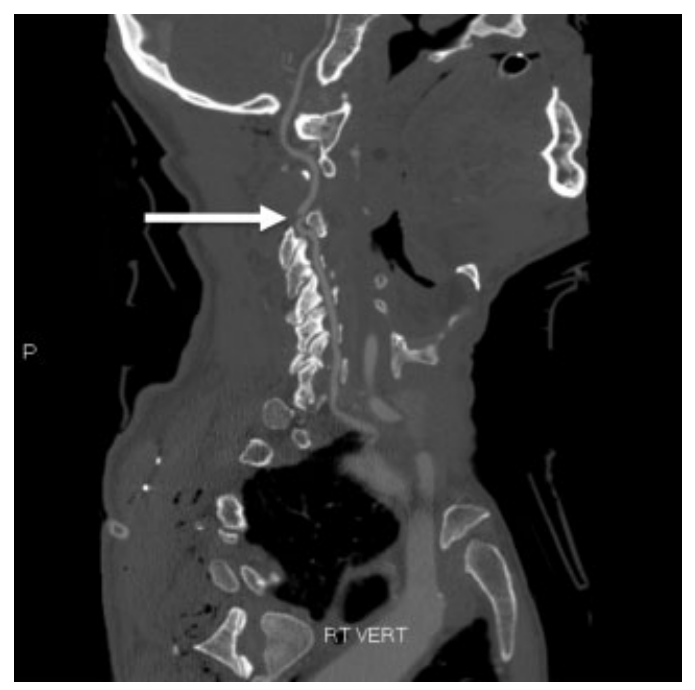

Fig. 5 Immediate postoperative sagittal CT angiographic image demonstrating patency across the VA injury repair site. CT, computed tomography; VA, vertebral artery. 


\section{Acknowledgments}

Analytic support for this work was provided by Spectrum Research, Inc. with funding from AOSpine.

\section{Disclosures}

The University of Rochester Spinal Surgery Fellowship has received AOSpine Funding for the years 2010 to 2013.

\section{References}

1 Wright JG, Swiontkowski MF, Heckman JD. Introducing levels of evidence to the journal. J Bone Joint Surg Am 2003;85-A(1):1-3

2 Methods Guide for Effectiveness and Comparative Effectiveness Reviews. AHRQ Publication No. 10(12)-EHC063-EF. Rockville, MD. Available at: www.effectivehealthcare.ahrq.gov. Accessed 2012

3 West S, King V, Carey TS, et al. Systems to Rate the Strength of Scientific Evidence. Evidence Report/Technology Assessment No. 47 (Prepared by the Research Triangle Institute-University of North Carolina Evidence-based Practice Center, Contract No. 290-97-0011). Rockville, MD: Agency for Healthcare Research and Quality; 2002

4 Atkins D, Best D, Briss PA, et al; GRADE Working Group. Grading quality of evidence and strength of recommendations. BMJ 2004; 328(7454):1490

5 Eskander MS, Drew JM, Aubin ME, et al. Vertebral artery anatomy: a review of two hundred fifty magnetic resonance imaging scans. Spine (Phila Pa 1976) 2010;35(23):2035-2040

6 Hong JT, Park DK, Lee MJ, Kim SW, An HS. Anatomical variations of the vertebral artery segment in the lower cervical spine: analysis by three-dimensional computed tomography angiography. Spine (Phila Pa 1976) 2008;33(22):2422-2426

7 Oga M, Yuge I, Terada K, Shimizu A, Sugioka Y. Tortuosity of the vertebral artery in patients with cervical spondylotic myelopathy. Risk factor for the vertebral artery injury during anterior cervical decompression. Spine (Phila Pa 1976) 1996;21(9):1085-1089

8 Sasso RC, Smucker JD, Hacker RJ, Heller JG. Artificial disc versus fusion: a prospective, randomized study with 2-year follow-up on
99 patients. Spine (Phila Pa 1976) 2007;32(26):2933-2940, discussion 2941-2942

$9 \mathrm{Lu}$ J, Wu X, Li Y, Kong X. Surgical results of anterior corpectomy in the aged patients with cervical myelopathy. Eur Spine J 2008; 17(1):129-135

10 Pechlivanis I, Brenke C, Scholz M, Engelhardt M, Harders A, Schmieder K. Treatment of degenerative cervical disc disease with uncoforaminotomy-intermediate clinical outcome. Minim Invasive Neurosurg 2008;51(4):211-217

11 Shen FH, Samartzis D, Khanna N, Goldberg EJ, An HS. Comparison of clinical and radiographic outcome in instrumented anterior cervical discectomy and fusion with or without direct uncovertebral joint decompression. Spine J 2004;4(6):629-635

12 Bertalanffy H, Eggert HR. Complications of anterior cervical discectomy without fusion in 450 consecutive patients. Acta Neurochir (Wien) 1989;99(1-2):41-50

13 Bilbao G, Duart M, Aurrecoechea JJ, et al. Surgical results and complications in a series of 71 consecutive cervical spondylotic corpectomies. Acta Neurochir (Wien) 2010;152(7): $1155-1163$

14 Burke JP, Gerszten PC, Welch WC. Iatrogenic vertebral artery injury during anterior cervical spine surgery. Spine J 2005;5(5):508-514, discussion 514

15 Busch G. Anterior fusion for cervical spondylosis. J Neurol 1978; 219(2):117-126

16 Choi G, Lee SH, Bhanot A, Chae YS, Jung B, Lee S. Modified transcorporeal anterior cervical microforaminotomy for cervical radiculopathy: a technical note and early results. Eur Spine J 2007; 16(9):1387-1393

17 Fountas KN, Kapsalaki EZ, Nikolakakos LG, et al. Anterior cervical discectomy and fusion associated complications. Spine (Phila Pa 1976) 2007;32(21):2310-2317

18 Graham AW, Swank ML, Kinard RE, Lowery GL, Dials BE. Posterior cervical arthrodesis and stabilization with a lateral mass plate. Clinical and computed tomographic evaluation of lateral mass screw placement and associated complications. Spine (Phila Pa 1976) 1996;21(3):323-328, discussion 329

19 Katonis P, Papadakis SA, Galanakos S, et al. Lateral mass screw complications: analysis of 1662 screws. J Spinal Disord Tech 2011; 24(7):415-420

\section{Editorial Perspective}

Our EBSJ reviewers agreed with the importance of the topic, the validity of the key questions asked and the methodology employed for the underreported subjects of vertebral artery (VA) anomaly and VA injury in spinal surgery. The aims were (1) to assess the reliability of classification systems of VA anomaly and (2) to determine the incidence of VA injury and the treatments that were successful.

The authors were prompted to perform this systematic review in light of the apparent paucity of published literature regarding VA injury and treatment outcomes particularly. There is agreement that intraoperative VA injury is a life threatening and likely underreported complication in cervical spinal surgery. The review highlights the increased risk of VA injury with corpectomy compared with anterior cervical discectomy and fusion and, moreover, injury to the usually dominant left VA. The authors recommend preoperative computed tomography angiography or magnetic resonance angiography to exclude VA anomalies and to recognize the risk of intraoperative VA injury, especially in older patients with tortuous vessels.

In terms of treatment, the authors identify generally poor results with tamponade alone and recommend further angiographic studies. Primary repair and ligation is described as feasible in posterior cervical approaches, but embolization is preferable in anterior VA injuries. The single case series by Bilbao et al (2010) reporting embolization is noted but embolization is becoming the standard of care in contemporary clinical practice.

The reviewers noted several substantial deficiencies to this study: In terms of surgical intervention, ligation is not the same as repair, whether this is done endoluminally or at open surgery. Obviously, ligation has the risk of hypoperfusion and stroke, especially when performed on the dominant VA. The ideal is to maintain flow patency while restoring mural continuity (specifically closing the hole without ligating the 
vessel). It should be pointed out that open surgical ligation should be preferably performed rostrally and distally to assure hemostasis and limit "backflow," which adds to the surgical challenge.

Endoluminal options receive only minimal discussion in this review and include coil occlusion of the VA above and below the injury to occlude the VA, embolization of a false aneurysm in an attempt to maintain patency and the deployment of a covered stent to exclude the injury while maintaining patency.

Our reviewers were in disagreement with the authors' conclusion that surgical repair of VA injuries is the treatment of choice. This conclusion appeared to be based on two successful repairs reported by Burke et al in 2005, in addition to two further reports of ligation. There was no discussion of the access/location of the injured VA injured. In fact, the variability of VA injuries makes any generalization toward open surgical intervention very problematic. Surgical access to the VA injury site while maintaining local bleeding control can be very challenging. If the VA is not in the canal and can be exposed from a laterally expanded approach then repair by suture to maintain VA patency is feasible and appears prefer- able. If the arterial injury is in the bony canal, access for repair requires extensive removal of the lateral vertebral body to facilitate adequate exposure. Another important consideration concerning open surgical ligation is the use of clips. The potential for nerve root injury should be contemplated as these are placed. Open surgical ligation also prevents stenting of the VA should recannulization be desired, for instance, in case of a dominant side injury.

In conclusion, this article provides a valuable overview of the current state of our knowledge base on this underreported subject of VA injuries. Hopefully, increased awareness of VA anatomy and variations will further decrease intraoperative injury risks. Knowledge of options regarding repair, embolization, and ligation is helpful but at this point in time, EBSJ finds that there is insufficient evidence to suggest a specific treatment. Future, larger investigations are likely to change our understanding and lead to different treatment recommendations. The entity of VA would be an ideal example for installation of a fiduciary databank, for instance, operated by AOSpine. Such a databank could allow for much improved understanding of our management of intraoperative VA injuries. 